\title{
Laboratory Animal Science
}

National Cancer Institute

\section{Source}

National Cancer Institute. Laboratory Animal Science. NCI Thesaurus. Code C16776.

The science and technology concerned with the procurement, selection, health and welfare of animals used in research and testing. 